\title{
LA TEORÍA DE LA JUSTICIA DE NANCY FRASER COMO CONTRIBUCIÓN AL ANÁLISIS DE LAS POLÍTICAS DE ACCIÓN AFIRMATIVA EN LA EDUCACIÓN SUPERIOR BRASILEÑA
}

\section{ARTÍCULO ORIGINAL}

GUIMARÃES, Matheus De Oliveira ${ }^{1}$

GUIMARÃES, Matheus De Oliveira. La teoría de la justicia de Nancy Fraser como una contribución al análisis de las políticas de acción afirmativa en la educación superior brasileña. Revista Científica Multidisciplinar Núcleo do Conhecimento. año 04, Ed. 11, Vol. 01, págs. 22-38. Noviembre de 2019. ISSN: 2448-0959, Enlace de acceso: https://www.nucleodoconhecimento.com.br/educacion-es/teoria-justicia

\section{RESUMEN}

Este artículo busca la presentación de la formulación de la justicia social designada por la filósofa política estadounidense Nancy Fraser a través de la cual ella interpreta el escenario social hoy a partir del reconocimiento-redistribución binomial. Presentaremos argumentos teóricos que pueden ser de interesante valor para el análisis, interpretación y evaluación de las políticas educativas brasileñas directamente relacionadas con el tema de las políticas de acción afirmativa. A partir de la presentación de la teoría de Frasean sobre justicia social y reconocimiento, buscamos relacionar este pensamiento, de manera panorámica, con las acciones afirmativas dirigidas a la educación superior implementadas en Brasil, especialmente

\footnotetext{
${ }_{1}^{1}$ Estudiante de doctorado en El derecho a la educación y las políticas educativas del Programa de Posgrado en Educación de la Pontificia Universidad Católica de Minas Gerais (PucMinas). Máster en Educación, vinculado a la línea de investigación Diversidad, Inclusión y Prácticas Educativas, por la Universidad Federal de Ouro Preto (UFOP). Es licenciado en Derecho (UFOP), Filosofía (ICSH) y Pedagogía (FGD), especializado en Prácticas Pedagógicas (UFOP) y Gestión de Políticas Públicas (UFOP).
} 
desde la década de 1990. Con este fin, se demuestran los principales argumentos de Nancy Fraser, para los que la justicia exige hoy tanto la redistribución de los bienes sociales y la riqueza como el reconocimiento cultural-valorativo de las diferencias. Por último, se establece la relación teórica entre las acciones afirmativas y la teoría fraseana.

Palabras clave: acciones afirmativas, educación superior, justicia social, Nancy Fraser, raza, redistribución-reconocimiento.

\section{INTRODUCCIÓN}

La Constitución brasileña de 1988, en su artículo 206, punto I, y la Ley de Directrices y Bases de Educación de Brasil de 1996, en el artículo 3, punto I, establecen que la enseñanza debe ser provisión respetando el principio de igualdad de condiciones. En consonancia con este precepto constitucional, las políticas educativas comenzaron a formularse e implementarse con el propósito de reducir las desigualdades sociales que han prevalecido en la historia de Brasil, discriminando a diversos segmentos de la sociedad.

En las últimas dos décadas, con respecto al tratamiento del tema racial en Brasil, se han producido cambios significativos en el país que han estado produciendo intensas transformaciones en la sociedad. En este contexto, se incluyen acciones afirmativas, entendidas como iniciativas para promover la inclusión social y, al mismo tiempo, se valoran aspectos singulares de grupos e individuos.

La conexión entre las discusiones de Educación, Derecho y Ciencias Políticas con temas relacionados con temas relacionados con cuestiones de redistribución y reconocimiento - y específicamente con respecto a la raza - se basa significativamente en su énfasis incuestionable en el comprender y hacer frente a las desigualdades en el contexto social, tanto en las relaciones de poder como en el conocimiento, inelutably interconectados (APPLE; BOLA; GANDIN, 2013; GILLBORN; LADSON-BILLINGS, 2013; APPLE, 2017). A pesar de la comprensión de muchos de un supuesto carácter epifenomena de lo que eventualmente se referiría a las 
discusiones sobre la clase y el capitalismo, es esencial entender la fuerza relativamente autónoma de las cuestiones de raza y la importancia de las particularidades de la vida cotidiana de quienes viven tales situaciones (APPLE, 2017).

En este sentido, con la necesidad de proporcionar acceso (y permanencia) a la educación superior a quienes han descuidado sus posibilidades de educación educativa, las políticas públicas brasileñas han estado buscando una universalización tardía de la educación superior y, posiblemente a pesar de lo previsto, terminan en línea con la pérdida de la calidad de la enseñanza sin democratizar efectivamente el acceso. Aunque en las últimas dos décadas los indicadores educativos han mejorado para todos los rangos de enseñanza y en todos los grupos sociales, todavía se observan graves desigualdades raciales, especialmente en las primeras etapas (HEILBORN; ARA-JO; BARRETO, 2010).

A pesar del debate sobre las políticas de acción afirmativa se ha centrado, especialmente desde la última década, en el sistema de cuotas para las universidades públicas, las acciones del Estado implican acciones que van más allá de las cuotas, con características y alcance diferenciado.

Aunque es posible afirmar que en el gobierno Fernando Henrique Cardoso ya había iniciativas federales dirigidas a la población negra, el análisis de los documentos de la época revela que la estrategia discursiva y la política de dicho gobierno era promover el reconocimiento sin inversiones en el aspecto redistributivo, aunque la desigualdad fue la principal justificación de las políticas de valoración de la población negra (LIMA, 2010).

El comienzo de la administración de Lula en 2003 marca un cambio denso en las políticas con una perspectiva racial. Como Lima (2010) afirma, hasta entonces la relación del estado con el Movimiento Negro era de exterioridad, constituyendo básicamente actores sociales como demandantes y con poca inserción en el aparato gubernamental. En el gobierno de Lula, esta relación se convierte en la incorporación, 
en sus marcos, de los representantes negros (dando mayor visibilidad a las demandas del movimiento).

El hecho es que el tema de la educación superior es sin duda el que moviliza el debate más público sobre las acciones afirmativas, especialmente en lo que respecta a la implementación de políticas de cuotas en las instituciones públicas de educación superior y los análisis sobre los estudiantes los titulares de cuotas. Sin embargo, como lima (2010) afirma, la marcada reacción social a la política de cuotas no tuvo la misma repercusión cuando el gobierno implementó un programa de acciones afirmativas en el sistema privado, responsable de más del $80 \%$ de la matrícula en la educación superior brasileña .

Las principales políticas públicas del ámbito federal con recorte racial en la educación se organizan hoy tanto en el ámbito del reconocimiento cultural como en el de la redistribución. En Brasil, además de la infame política de cuotas (a menudo erróneamente entendida como una síntesis de acciones afirmativas), PROUNI (Programa Universidad para Todos), a través del cual el Gobierno Federal ofrece becas (integrales y para los estudiantes de bajos ingresos para que puedan estudiar en universidades privadas; (Fondo de Financiación Estudiantil), un programa del Ministerio de Educación destinado a financiar la educación de pregrado de los estudiantes matriculados en instituciones no libres; y el PBP (Permanence Scholarship Program), una ayuda financiera (ofrecida por el Gobierno Federal a estudiantes de bajos ingresos en universidades públicas federales y a estudiantes con becas completas de PROUNI) que tiene como objetivo minimizar las desigualdades sociales y contribuir a la permanencia y graduación de los estudiantes de pregrado en una situación de vulnerabilidad socioeconómica.

De estas políticas, Prouni es, por supuesto, la acción afirmativa más redistributiva de mayor impacto en la historia de la educación superior en el país. Creada en 2004, bajo el marco de Lula, por la Medida Provisional 213/2004, Prouni fue institucionalizada en 2005 por la Ley 11.096/2005. Este programa tiene como objetivo otorgar becas completas y parciales a estudiantes de bajos ingresos en cursos de pregrado y 
secuenciales de formación específica en instituciones privadas de educación superior. Se ofrecen exenciones fiscales a las instituciones alas del Programa.

Corroborando la idea de la democratización truncada del acceso a la educación superior a partir de acciones afirmativas, Predijo Pereira, refiriéndose indirectamente a los resultados de Prouni:

[...] el sector privado en Brasil p[responde]or el $88 \%$ de la oferta de vacantes y por el $71 \%$ de la matrícula, lo que lo convierte en uno de los más grandes del mund[...]o. Con la ayuda de la red privada, la oferta de vacantes para la educación superior cubre hoy el $86 \%$ de los graduados de secundaria. [Contudo][...]sólo el $10 \%$ de los jóvenes de dieciocho a veinticuatro años asisten a ella (2009, p. 45).

Pereira (2009) añade que las vacantes ociosas en HEI privado eran, antes de la implementación de Prouni, en $49.5 \%$ - mientras que en los HEl federales esta tasa era del $0,9 \%$ y, en el estado, del $4,7 \%$.

Sobre las políticas de expansión de la educación superior, Pereira informa:

El escenario económico que estamos experimentando está demostrando que los efectos de la democratización educativa no conducen a la democratización social, sino que pueden producir nuevas y más dolorosas desigualdades. (2009, p. 46).

Y es exactamente como una propuesta para el estudio de este contexto complejo que indica el análisis de las teorías presentadas por Nancy Fraser (2008, 2003, 2002, $2000,1997)$. ¿Serían eficaces para promover la transformación social las acciones afirmativas encaminadas a democratizar el acceso y la permanencia de las minorías en la educación superior? Desde el punto de vista económico, ¿serían los mismos efectivos? ¿Y cuáles son sus impactos en el campo del reconocimiento cultural de su público objetivo?

No se pretende, en este texto, respuestas concluyentes a estas preguntas. Sin embargo, se cree que la comprensión de los argumentos fraseos en este contexto es de un valor interesante para este análisis. 


\section{POLICIAS ECONOMICAS-DISTRIBUTIVAS $X$ POLICIAS DE} VALORACION CULTURAL: TEORIZACIONES DE NANCY FRASER SOBRE EL DILEMMA REDISTRIBUTION-RECOGNITION COMO [2]UN SUBSIDIO PARA LA INTERPRETACIONDE LA POLICIA DE ACCIONES AFIRMATIVAS EN BRASILIA HIGHER EDUCATION

Nancy Fraser $(2008,2003,2002,2000,1997)$ señala la lucha por el reconocimiento como algo que se convierte, en zancadas, en la forma paradigmática de conflicto político desde finales del siglo pasado. Según Fraser (2008, 2003, 2002, 2000, 1997), las demandas de reconocimiento de la diferencia alimentan las luchas de grupos movilizados bajo diferentes banderas: nacionalidad, etnia, raza, género y sexualidad. En consecuencia, la identidad grupal va más allá, en estos conflictos (llamados postsocialistas), el interés de clase como el principal medio de movilización política: "La domi[a]nación cultural sustituye a la explotación como injusticia fundamental. Y el reconocimiento cultural toma el lugar de la redistribución socioeconómica como remedio para la injusticia y el objetivo de la lucha política" (FRASER, 2008, p.11, traducción mía).

Sin embargo, esto no es todo, ya que las luchas por el reconocimiento se desarrollan en un mundo de desigualdad material exacerbada, especialmente en los países del sur.

En este contexto, en sus teorías, Fraser $(2008,2003,2002,2000,1997)$ plantea algunas cuestiones que esbozarán su debate sobre los dilemas de la justicia:

Entonces, ¿cómo deberíamos ver el eclipse de un imaginario socialista centrado en términos como interés, exploración y redistribución? ¿Y qué debemos hacer con el surgimiento de una nueva imaginería política centrada en las nociones de identidad, diferencia, dominación cultural y reconocimiento? ¿Esta guiada representa un lapsus de falsa conciencia? O, en cambio, ¿sería una manera de compensar la ceguera cultural de un paradigma marxista puesto en desgobierno por el colapso del comunismo soviético? (FRASER, 2008, p.11, traducción mía, grifos de autor). 
Categóricamente, Fraser $(2008,2003,2002)$ afirma que ninguna de estas posiciones sería apropiada, matizándolas como "demasiado completas y sin matices". En lugar de simplemente respaldar o rechazar lo que sería simple en la política de identidad, debe considerarse que hay un desafío intelectual y práctico por delante: el de desarrollar una teoría crítica del reconocimiento que identifique y asuma la defiende únicamente las versiones de la política cultural de diferencia que pueden combinarse de conformidad con la política de igualdad social (FRASER, 2008, p. 11-12).

Al formular este proyecto, Fraser (2008) afirma que la justicia hoy requiere tanto redistribución como reconocimiento, proponiendo examinar la relación entre ambos. Esto, según el autor, significaría, en parte, pensar en cómo conceptualizar e/ reconocimiento cultural y la igualdad social de una manera que ambos se sostengan a sí mismos- en lugar de aniquilarse (ya que hay varias concepciones que compiten entre ellos). Además, también significaría teorizar sobre los medios a través de los cuales la privación económica y la falta de respeto cultural se entrelazan y se apoyan mutuamente de forma concomitante. En este sentido, además, para el autor, la justicia también exige que se aclaren los dilemas políticos que surgen cuando tratamos de combatir ambas injusticias al mismo tiempo (FRASER, 2008, p. 12).

Fraser alega, como uno de sus propósitos:

(...) dos problemas políticos que ahora se desvinculan entre sí. Sólo con la integración entre reconocimiento y redistribución llegaremos a un marco conceptual adecuado a las demandas de nuestra era (FRASER, 2008, p.12, traducción mía).

Fraser presenta dos modos genéricos de comprensión de la injusticia (2008, p. 1318).

La primera sería la que se refiere a la injusticia económica, que, según ella, está arraigada en la estructura económico-política de la sociedad (explotación, marginación económica y privación) y cuya conceptualización de su naturaleza ya se ha llevado a cabo, a través de grandes esfuerzos, por parte de teóricos igualitarios (FRASER, 2008, p. 13-14). 
La segunda manera de entender la injusticia, según Fraser (2008), es cultura/ o simbólica, profundizando la injusticia en los patrones sociales de representación, interpretación y comunicación, cuyos ejemplos, según el autor, incluyen la dominación cultural (sobre la sumisión a patrones de interpretación y comunicación asociados con otra cultura, no relacionada y/o hostil a la suya); ocultación (relacionada con la invisibilización por el efecto de prácticas comunicativas, interpretativas y de representación consentidas por la propia cultura); y la falta de respeto (caracterizado por la difamación y la descalificación rutinaria en representaciones culturales públicas estereotipadas y/o en las interacciones de la vida cotidiana) (FRASER, 2008, p. 14).

Sin embargo, a pesar de las distinciones entre estas dos formas de injusticia (socioeconómica y cultural), Fraser (2008, 2003, 2002, 2000) afirma que ambas están marcadamente presentes en las sociedades contemporáneas. Ambos están arraigados en procesos y prácticas que promueven desventajas para algunos grupos en comparación con otros. En este sentido, tanto una como la otra forma de injusticia deben ser remediadas, aunque de diferentes maneras.

Para Fraser $(2008,2003,2002,2000)$, el recurso a la injusticia económica sería una especie de reestructuración político-económica que podría implicar la redistribución de los ingresos, la reorganización de la división de la transformación de otras estructuras económicas básicas. El filósofo político atribuye a este conjunto de remedios para la injusticia económica el término genérico redistribución.

En cambio, según el autor, el remedio para la injusticia cultural sería algún tipo de cambio cultural o simbólico. Esto podría implicar valorar las identidades irrespetadas (así como los productos culturales de los grupos difamados), la apreciación positiva de la diversidad cultural y, más radicalmente, podría abarcar una transformación integral de las normas sociales representación, interpretación y comunicación, con el fin de transformar el significado del yo y de todas las personas. Fraser $(2008,2003$, 2002, 2000) da a este conjunto de recursos para la injusticia cultural el término reconocimiento genérico. 
Para el autor (FRASER, 2008), cuando se trata de colectividades que se acercan al tipo ideal de la clase explotada, se enfrentan injusticias distributivas que carecen de remedios redistributivos (cuya lógica sería promover la homogeneización y consecuente caracterización errónea de los grupos). Cuando, a su vez, nos ocupamos de colectividades que se acercan al tipo ideal de sexualidad despreciada, por ejemplo, se enfrentan injusticias negativas a la discriminación que necesitan remedios de reconocimiento (cuya lógica, a su vez, sería la valorización de la miembro del reconocimiento de sus especificidades). Sin embargo, a medida que se aleja de los extremos, las cosas se vuelven borrosas y la diferenciación se vuelve mucho más compleja y delicada. Según Fraser (2008), al considerar las colectividades ubicadas en la región intermedia del espectro conceptual, existen tipos híbridos que mezclan características de la clase explotada con características de sexualidad despreciada. Estas colectividades se llaman, como el autor predice, bivalentes. Se diferencian como colectividades tanto por la estructura económica política como por la estructura cultural-valorativa de la sociedad:

Estas colectividades bivalentes (oprimidas o subordinadas) sufren injusticias que simultáneamente se remontan a la economía política y la cultura. En resumen, como informa Fraser, estas colectividades pueden sufrir de mala distribución socioeconómica y desprecio cultural, de modo que ninguna de estas injusticias es, en sí misma, un efecto indirecto del otro, pero tanto primario como cooriginal. En este caso, ni los medicamentos de redistribución ni los de reconocimiento por sí solos serían suficientes. Fraser afirma categóricamente: "las colectividades bivalentes carecen de amb[redistribuição e reconhecimento]as" (FRASER, 2008, p. 23, mi traducción).

Dependiendo de Fraser, el género y la raza son paradigmas de colectividades bivalentes. Aunque tienen particularidades no compartidas entre sí, ambas abarcan dimensiones económicas y culturales-valorativas: "género y raza, por lo tanto, implican tanto redistribución como reconocimiento" (FRASER, 2008, p. 23, traducción de la mina). 
Por lo tanto, según Fraser (2008, p. 25, mi traducción), "la raza[...] es un modo bivalente de la colectividad".

Por un lado, la lucha contra el racismo se asemeja a la clase, siendo un principio estructural de la economía política. En este sentido, la raza estructura la división capitalista del trabajo. Ocupaciones mal pagadas y de bajo estatus, aquellas aburridas, sucias y domésticas están ocupadas desproporcionadamente por ${ }^{[3]} \mathrm{OS}$ negros, mientras que esas ocupaciones mejor pagadas, con mayor estatus, cuello blanco, profesionales, técnicas y están ocupados en su mayoría por blancos.

Además, según el autor $(2008,2003,2002,2000,1997)$, la raza también estructura el acceso al mercado laboral formal, constituyendo grandes segmentos de la población negra como subclase (ignorada incluso para la propia explotación) y excluida del sistema Productivo. Como resultado, existe una estructura político-económica que genera modos de explotación, marginación y privación marcados específicamente por la raza: "esta estructura constituye la raza como una diferenciación económico-política que terminó con características" (2008, p. 26, mi traducción).

A este respecto, según el autor (2008), la injusticia racial aparece como una especie de injusticia distributiva que exige una compensación redistributiva. Por lo tanto, de una manera muy similar a la clase, la justicia racial requiere la transformación de la economía política para eliminar esta racialización. Y la lógica del remedio es similar a la lógica relacionada con la clase: "se trata de hacer carrera fuera del negocio" (FRASER, 2008, p. 26, mi traducción). Si la raza no es más que una diferenciación económico-política, la justicia requiere, como dice Fraser (2008, 2003, 2002, 2000), que sea abolida como criterio de diferenciación, en este sentido.

Sin embargo, como dijo el autor cuando se trata de género, la raza, así como eso, no es sólo económico-político. La raza también tiene dimensiones culturales-valorativas que la insertan en el universo de reconocimiento, abarcando también elementos más similares a la sexualidad que con la clase (FRASER, 2008, p. 26). 
Como señala Fraser (2008), un aspecto basal del racismo es el eurocentrismo caracterizado por la construcción autorizada de normas que favorecen los rasgos asociados con la branquitude. A esto se añade el racismo cultural, caracterizado, a su vez, por la descalificación generalizada de las cosas codificadas como negras, marrones o amarillas (pág. 26).

Como en el caso del género, estos daños son injusticias de reconocimiento y, por lo tanto, la lógica del recurso es también otorgar un reconocimiento positivo a un grupo específicamente devaluado.

Fraser $(2008,2003,2002,2000,1997)$ señala así la raza como un modo bivalente de colectividad, con un rostro económico-político y otros culturales-valorativos - ambos rostros entrelazados para fortalecerse mutuamente, dialécticamente - más aún porque el las normas culturales racistas y eurocéntricas se institucionalizan en el Estado y en la economía, mientras que la desventaja económica que sufren los negros restringe su voz.

Para compensar la injusticia racial, la economía política y la cultura se mueven. Pero, como lo demuestra el autor al tratar con el género, el carácter bivalente de la raza es también la fuente de un dilema.

Fraser $(2008,2003,2002,2000)$ afirma que el género y la raza son, por lo tanto, modos dileméticos de colectividad. A diferencia de la clase, que ocupa un extremo del espectro conceptual presentado por el autor, y la sexualidad, que ocupa la otra punta, el género y la raza son bivalentes, siendo implicados simultáneamente en la política de redistribución y en la política de la Reconocimiento.

Pero, ¿cómo podría disolverse la diferenciación al mismo tiempo y disolverse la especificidad de una colectividad despreciada?

Después de presentar el dilema de la redistribución-reconocimiento de una manera aparentemente intratable y de asumir que los remedios redistributivos para la injusticia económico-política siempre diferencian a los grupos sociales, mientras que los remedios de reconocimiento la injusticia cultural-valorizante siempre pone de relieve 
la diferenciación del grupo social, Fraser (2008, p. 28) propone examinar concepciones alternativas de redistribución -por una parte- y reconocimiento- por otro.

Mediante remedios afirmativos para la injusticia, Fraser entiende los que tienen por objeto corregir los efectos desiguales de los acuerdos sociales sin la estructura subyacente que los engendra (2008, p. 28).

Por remedios transformadores, en cambio, el autor comprende los centrados en corregir los efectos desiguales mediante la remodelación de la estructura gerativa subyacente (2008, p. 28).

En cuanto al autor, el punto fundamental de contraste entre los remedios afirmativos y los recursos transformadores para la injusticia es "efectos terminales frente a procesos que los producen. No es un cambio gradual frente al cambio apocalíptico" (FRASER, 2008, p. 28, mi traducción).

Fraser (2008) explica esta distinción, en primer lugar, en el caso de los recursos para la injusticia cultural. Según la autora, los remedios afirmativos para tales injusticias están asociados con lo que ella llama "multiculturalismo convencional", una especie de multiculturalismo que propone compensar la falta de respeto por la revaluación de las identidades de grupo injustamente devaluado (dejando intacto el contenido de estas identidades y las diferencias de grupo subyacentes). Por el contrario, los remedios transformadores se asocian con la deconstrucción, compensando la falta de respeto a través de la transformación de la estructura cultural-valorativa subyacente. Como afirma Fraser, desestabilizando las identidades y diferenciaciones de los grupos existentes, estos remedios no sólo elevarían la autoestima de los miembros de grupos que desrespetan recurrentemente, sino que también transformarían el sentido de pertenencia y afiliación de todos (pág. 28-29).

Para el autor, la cuestión no se trata de la disolución de todas las diferencias en una identidad humana única y universal, sino más bien el mantenimiento de un campo de múltiples diferencias, no binaria, fluida, siempre en movimiento: 
Si bien los remedios de reconocimiento afirmativo tienden a promover las diferencias de grupo existentes, los remedios de reconocimiento transformador transformador tienden a largo plazo para desestabilizarlos a fin de dar lugar a reagrupaciones futuras (FRASER, 2008, p. 30, mi traducción).

Se aplican distinciones similares a los recursos para la injusticia económica. Como dice Fraser $(2008,2003,2002)$, los remedios afirmativos para este tipo de injusticia están históricamente asociados con el estado de bienestar liberal, buscando, a través de ellos, una compensación por la mala distribución de la terminal (dejando la mayor parte de la estructura económico-política subyacente). Los remedios transformadores, en contra, se asocian históricamente con el socialismo, tratando de compensar la distribución injusta a través de la transformación de la estructura económico-política existente.

Fraser afirma que un enfoque destinado a compensar las injusticias en la distribución puede terminar creando injusticias de reconocimiento (2008, p. 31).

La redistribución afirmativa presupone una concepción universalista del reconocimiento (igual valor moral de las personas; titulada por Fraser (2008, 2003, 2002) como compromiso formal con el reconocimiento). Sin embargo, la práctica de la redistribución afirmativa, si se prolonga, tiende a poner en marcha una dinámica secundaria de estigmatización del reconocimiento que contradice su propio compromiso formal con el universalism ${ }^{[4]} \mathrm{O}$. El autor (2008), contrastando esta lógica con remedios transformadores para las injusticias distributivas de clase, predice que combinan programas universalistas de bienestar social, altos impuestos, políticas macroeconómicas destinadas a crear pleno empleo, un vasto sector público no mercado, importantes propiedades públicas y/o colectivas, y decisiones democráticas con respecto a las prioridades socioeconómicas básicas. Los remedios transformadores de redistribución tienden a disolver la diferenciación de clases, reduciendo la desigualdad social sin crear clases estigmatizadas de personas vulnerables que se consideran beneficiarias de especial generosidad. Fraser afirma que estos recursos tienden a promover la reciprocidad y la solidaridad en las relaciones de reconocimiento, pudiendo así hacer frente a la compensación por las 
injusticias de redistribución para ayudar, también, a compensar ciertas injusticias reconocimiento (2008, p.31-33)

Fraser (2008) afirma que, al igual que la redistribución afirmativa, la redistribución transformadora (en general) presupone una concepción universalista del reconocimiento: el mismo valor moral de las personas. Sin embargo, a diferencia de la redistribución afirmativa, la práctica de la redistribución transformadora tiende a no disolver esta concepción.

A partir de esta discusión, después de considerar sólo las ideas típicas puras de casos en ambos extremos del espectro conceptual, y el contraste de los efectos divergentes de los remedios afirmativos y transformadores a las injusticias distributivas arraigadas económicamente - por un lado - y los de reconocimiento culturalmente arraigado por el otro - se ve que: 1 . los remedios afirmativos generalmente tienden a promover la diferenciación de grupo, mientras que los remedios transformadores tienden a desestabilizar y difuminar esta diferenciación; y 2. Los remedios afirmativos de redistribución pueden generar una protesta de desprecio, mientras que los remedios transformadores de redistribución pueden ayudar a compensar algunas formas de no reconocimiento. En este sentido, Fraser $(2008,2003,2002)$ sugiere una manera de reformular el dilema de la redistribución-reconocimiento.

Sin embargo, al concluir este debate, Fraser plantea el interrogatorio:

[...] con respecto a los grupos sometidos a ambos tipos de injusticia, ¿cuál sería la combinación de recursos que funcionaría mejor para minimizar (si no eliminar para siempre) las interferencias mutuas que surgen al buscar la redistribución y el reconocimiento a la a la misma hora? (2008, p. 33, mi traducción).

Y eso todavía debe ser problematizado. 


\section{CONSIDERACIONES FINALES: LA CONTEXTUALIZACION DEL DEBATE DE FRASEANO A LA REALIDAD DE LA EDUCACION MAYOR EN BRASIL}

Se afirma que Brasil, porque se caracteriza por numerosas desigualdades, de diferentes órdenes, está marcado como un modelo paradigmático para pensar en la aplicación de la teoría del fraseo. En este sentido, las teorizaciones de Fraser se proponen para el análisis del escenario educativo brasileño.

Como afirma Ferreira (2010), la modernidad, al llegar a Brasil, encontró un país con marcas de conservadurismo y tradicionalismo, una situación que, en cierto modo, sigue siendo hoy en día en diversos sectores sociales. En el Brasil, los prejuicios y la exclusión racial han tenido lugar (y aún conservan, en cierta medida) de manera velada, no institucionalizada y abiertamente reconocida. Esto compromete, en muchos casos, los procesos de toma de medidas por parte del gobierno. Acciones como las políticas de acción afirmativa se consideran repetidamente una afrenta a la igualdad de oportunidades, incluso si la desigualdad, la segregación y los prejuicios son visibles (FERREIRA, 2010).

Es en este sentido que el desempeño de las autoridades públicas con miras a reducir las desigualdades sociales puede entenderse a partir de las teorías de Fraser (2008, 2003, 2002, 2000) sobre el reconocimiento y la redistribución. Es esencial que las políticas de redistribución y reconocimiento no se contradigan entre sí y, además, se guíen por el respeto mutuo (APPLE, 2017, p. 32).

Las políticas de acción afirmativa forman parte de Fraser (2008, 2003, 2002), como remedio contra las injusticias sociales. Sin embargo, como predice Ferreira (2012), un punto importante a tener en cuenta en esta discusión se refiere al hecho de que, todavía desde una perspectiva frasea, las acciones afirmativas no serían garantías absolutas para modificar positivamente la estructura que genera desigualdades. Esto se debe a que, tal vez, a priori, sólo reconocen la necesidad de distribución a los grupos con discapacidades históricas, pero no necesariamente resuelven los 
impapasos estructurales que generan tales desigualdades. La expectativa sería que los beneficiados por estas políticas pudieran, a gran escala y a largo plazo, transmitir sus resultados de crecimiento socioeconómico y cultural de manera intergeneracional para que sus descendientes ya no necesiten políticas de este tipo para mantenerse en aumento o alcanzar niveles de ingresos y estatus similares a los de la población que históricamente han recibido estos derechos en Brasil.

\section{REFERENCIAS}

APPLE, Michael W. A educação pode mudar a sociedade?. Petrópolis: Vozes, 2017.

APPLE, Michael W.; BALL, Stephen J.; GANDIN, Luís Armando. Mapeamento da sociologia da educação: contexto social, poder e conhecimento. In: Sociologia da Educação: análise internacional. Michael W. Apple; Stephen J. Ball; Luís Armando Gandin (orgs.). Porto Alegre: Penso, 2013.

LIMA, Márcia. Ações afirmativas no governo Lula. In: Revista Novos Estudos. 2010.

FERREIRA, Wallace. Justiça e reconhecimento em Nancy Fraser. Teresina: Jus Navigandi, 2012.

FRASER, Nancy. From Redistribution to Recognition? Dilemmas of Justice in a "Postsocialist" Age. In. Adding Insult to Injury. Nancy Fraser debates her critics. Edinburgh: Verso, 2008.

Justice Interruptus: Critical Reflections on the "Postsocialist" Condition. London: Routledge, 1997.

Redistribuição ou reconhecimento? Classe e status na sociedade contemporânea. In: Interseções - Revista de Estudos Interdisciplinares. UERJ, ano 4, n.1, 2002.

Recognition without Ethics? Theory, Culture \& Society, v. 18, p. 21-42, 2001. 
Da redistribuição ao reconhecimento? Dilemas da justiça na era pós-socialista. In: Democracia hoje: novos desafios para a teoria democrática contemporânea. Jessé Souza (org.). Brasília: Editora Universidade de Brasília, 2000.

FRASER, N.; HONNETH, A. Redistribution or recognition?: a political philosophical exchange. London; New York: Verso, 2003.

GILLBORN, David; LADSON-BILLINGS, Gloria. Educação e Teoria Racial Crítica. In: Sociologia da Educação: análise internacional. Michael W. Apple; Stephen J. Ball; Luís Armando Gandin (orgs.). Porto Alegre: Penso, 2013.

heIlboRN, M. L.; ARAÚJO, L.; BARReto, A. (Orgs). Gestão de Políticas Públicas em Gênero e Raça: GPP-GeR, Módulo I. Rio de Janeiro: CESPE; Brasília: Secretaria de Políticas Públicas para as Mulheres. 2010.

PEREIRA, Elisabete Monteiro de Aguiar. A universidade da modernidade nos tempos atuais. In: Revista Avaliação, v. 14, n. 1, p. 29-52. Campinas, 2009.

ZONINSEIN, Jonas; FERES JÚNIOR, João. Ação Afirmativa e Desenvolvimento. In: FERES JÚNIOR, João \& ZONINSEIN, Jonas (Orgs.). Ação Afirmativa e Universidade: experiências nacionais comparadas. Brasília: Editora Universidade de Brasília, 2006.

2. Toma-se, aqui, como referência principal para a análise que se propõe, a seguinte obra: FRASER, Nancy. From Redistribution to Recognition? Dilemmas of Justice in a "Postsocialist" Age. In. Adding Insult to Injury. Nancy Fraser debates her critics. Edinburgh: Verso, 2008.

3. "People of color", nos termos de Fraser.

4. Nos termos de Fraser, essa dinâmica secundária (estigmatizante) pode ser entendida como o efeito de reconhecimento prático da redistribuição afirmativa (2008, p. 31-32).

Enviado: Octubre, 2019. 
Aprobado: Noviembre, 2019. 